\title{
A Comparative Study between Hyaluronic Acid and Corticosteroids for the Treatment of the Greater Trochanteric Pain Syndrome
}

\author{
Asunción Acosta Pereira, Berta Magallares López, Arturo Rodriguez de la Serna* \\ Unidad de Reumatología, Hospital Santa Creu i Sant Pau, Barcelona, Spain \\ Email: ${ }^{*}$ arodriguezs@santpau.cat
}

Received 6 May 2015; accepted 7 July 2015; published 10 July 2015

Copyright (C) 2015 by authors and Scientific Research Publishing Inc.

This work is licensed under the Creative Commons Attribution International License (CC BY).

http://creativecommons.org/licenses/by/4.0/

(c) (i) Open Access

\begin{abstract}
Objective: To date, there are no studies addressing the efficacy of hyaluronic acid (HA) injections at the trochanteric bursa in patients with greater trochanteric pain syndrome (GTPS). The objective of the study was to compare the efficacy and safety of HA to corticosteroid injections for the treatment of the GTPS. Methods: This prospective, randomized, two-arm trial involved 47 patients with unilateral or bilateral GTPS. Patients received an intra-bursal injection of $40 \mathrm{mg}$ triamcinolone acetonide plus $1 \mathrm{~mL}$ lidocaine, or of $60 \mathrm{mg}$ HA. Patients completed visual analog scales (VAS) and Likert scales to evaluate interference of pain with daily activity, recovery from pain, and treatment satisfaction. A non-inferiority analysis was also performed. Results: Mean VAS score for pain significantly decreased comparing baseline with 1, 3 and 6 months in both treatment groups. VAS score for pain on palpation was also significantly lower than baseline in both arms. No significant differences were found between groups. Analysis of Likert scales at the sixth month didn't detect statistically significant differences between treatment groups. The non-inferiority analysis showed that the treatment with HA was non-inferior to corticosteroids. No secondary adverse effects were found among the patients of both groups during the follow-up. Conclusions: The treatment with HA has demonstrated to be non-inferior to corticosteroids after 6 months of follow-up in patients with GTPS. Therefore, the treatment with HA may be considered an effective alternative therapeutic strategy to reduce pain in patients in whom the treatment with corticosteroids, or other therapies, have been unsuccessful or contraindicated.
\end{abstract}

\section{Keywords}

Hyaluronan, Greater Trochanteric Pain Syndrome, Glucocorticoids, Quality of Life

\footnotetext{
${ }^{*}$ Corresponding author.

How to cite this paper: Acosta Pereira, A., Magallares López, B. and de la Serna, A.R. (2015) A Comparative Study between Hyaluronic Acid and Corticosteroids for the Treatment of the Greater Trochanteric Pain Syndrome. Open Journal of Rheumatology and Autoimmune Diseases, 5, 57-61. http://dx.doi.org/10.4236/ojra.2015.53010
} 


\section{Introduction}

The greater trochanteric pain syndrome (GTPS) is a frequent condition characterized by the pain and reproducible tenderness at the greater trochanter region, buttock, and lateral thigh [1]. The GTPS affects about 10\% - 25\% of the general population, and represents the cause of assisting in primary care for 1.8 patients per 1000 per year, mainly women [2] [3]. Primary strategies of GTPS treatments include injections of corticosteroids (such as triamcinolone acetonide) with local anesthetics, physical therapy, ice, weight loss, nonsteroidal antiinflammatory drugs, or behavior modification [4]-[8]. An arthroscopic bursectomy is proposed when conservative treatment fails. The treatment with steroid-anesthetic injections has been demonstrated to produce a rapid improvement in the pain and disability of $60 \%-100 \%$ of patients. Indeed, a single corticosteroid injection has been shown to increase in 2.7-fold the number of patients being free of pain after 5 years since the injection [3] [6]. Alternative therapeutic agents are required for diabetic patients, in whom the injection with corticosteroids may produce the increase to glucose levels [9], or patients who are refractory, intolerant to conventional corticosteroids, or those who have not achieved a long-term benefit [1]. Intra-articular injections of hyaluronic acid (HA) have been well established for the treatment of knee osteoarthritis [10]. The beneficial effects of HA on the synovial fluid of joints and bursas derive from its high viscosity, its protective effects on articular cartilage and joints, by influencing the inflammatory process of the synovial tissue and promoting the healing and regeneration of the articular cartilage. In view of these effects for intra-articular injections, it seems reasonable to extrapolate the same effectiveness to intra-bursal injections for the treatment of GTPS. To date, and with the exception of just one recent study [11], there are no published studies addressing the efficacy of HA injections at the trochanteric bursa in patients with GTPS. Therefore, the objective of the study is to compare the efficacy and safety of HA to corticosteroid injections for the treatment of the GTPS.

\section{Patients and Methods}

This prospective, randomized, two-arm trial involved patients with unilateral or bilateral GTPS from the Hospital de la Santa Creu i Sant Pau (Barcelona, Spain). All patients signed the informed consent before participating in this study. The inclusion criteria were as follows: diagnosis established by physical examination (Rasmussen and Fano clinical criteria); experiencing symptoms that persist for at least 1 month despite treatment; not having received local infiltrations in previous 6 months to recruitment; and signing the written informed consent. The criteria for exclusion were: having any intra-articular disease in the coxofemoral joint or pain back associated to any degenerative disease. Patients were randomized into 2 treatment groups receiving an intra-bursal injection of: $40 \mathrm{mg}$ triamcinolone acetonide (Trigon Depot ${ }^{\circledR}$ ) plus $1 \mathrm{~mL}$ lidocaine (group 1), or $60 \mathrm{mg}$ HA depot (Suplasyn ${ }^{\circledR}$ 1-Shot, group 2). Patients were not permitted to receive non-steroidal anti-inflammatory drugs; however, they continued having the same analgesics that before recruitment. The main objective was to determine the effectiveness of treatment with HA after 1, 3 and 6 months regarding pain and the pain on palpation. The secondary objective was to evaluate the safety of the treatment by considering the appearance of adverse events related to the injection. Follow-up evaluations were performed at baseline, and after 1, 3 and 6 months of starting the treatment. In each visit to the specialist, patients completed a visual analog scale (VAS) to evaluate baseline pain and pain on palpation, and a Likert scale to determine the interference of pain with daily activity, the recovery from the pain, and the treatment satisfaction after 6 months of follow-up.

\section{Statistical Analysis}

Quantitative variables were expressed as the mean and the standard deviation (SD); whereas, qualitative one as absolute and relative frequencies. Regarding patients with bilateral GTPS, their clinical data used in the study only derived from the most symptomatic side. Analyses were performed per protocol. Comparisons between different variables were performed by using the Chi-square or Fisher's exact test, in qualitative variables, or the T-test, in the quantitative ones. The statistical significance was established when $\mathrm{P} \leq 0.05$. The evolution of VAS scores during the follow-up period between the two treatment groups was analyzed and compared by using a repeated measures analysis of variance (ANOVA). A non-inferiority analysis was performed to test the effectiveness decreasing the pain after 6 months of treatment with HA compared to corticosteroids. Non-inferiority was established when the lower limit of the confidence interval for the difference between groups was less than 1 point. All statistical analyses were performed using SPSS 18.0 software. 


\section{Results}

From 52 patients initially recruited in the study, 4 were excluded due to protocol violation and 1 due to loss of follow-up. Therefore, the total number of patients included in the study was 47, 22 from the group receiving corticosteroids and 25 from HA. The population was predominantly female (87.2\%), with a mean age of 67.83 year (SD 11.1) in the group of corticosteroids and 63.95 (SD 12.0) in the HA group. No differences between treatment groups were found regarding sex, age, or side of the disease. Both groups showed similar VAS scores for pain at baseline, mean 7.45 (SD 1.8) for the group of corticosteroids and 8.22 (SD 1.29) for the group of HA. However, the group receiving HA showed a significant $(\mathrm{P}=0.015)$ higher VAS score for pain on palpation at baseline (mean 8.86; SD 1.27) than the group with corticosteroids (mean 7.95; SD 1.17). No differences between groups were found regarding interference of pain with daily activity at baseline. The evolution of VAS score for pain during the follow-up period between the two groups is shown in Figure 1. Although there were no differences between groups, mean scores for pain during the study significantly decreased $(\mathrm{P}<0.001)$ comparing baseline with 1, 3 and 6 months of treatments. The evolution of VAS score for pain on palpation during the follow-up period between the two groups is shown in Figure 2. Similarly, no differences between groups were found; however, scores at 1,3 and 6 months of treatments were significantly lower $(P<0.001)$ than at baseline. No differences were found between treatment groups regarding Likert scale evaluating the interference of pain with daily activity, recovery from pain, and treatment satisfaction. The non-inferiority study showed that the treatment with HA is non-inferior to corticosteroids. No secondary adverse effects were found among the patients of both groups during the follow-up period.

\section{Discussion}

When primary conservative approaches fails, injections with corticosteroids and/or local anesthetics achieves a pain relief in $60 \%-100 \%$ of the cases [1] [4]-[8]. However, some patients experience persistence of symptoms after several months of starting the corticosteroids treatment, revealing other underlying etiologies, error in the diagnosis or in the location of the injection, or simply non-response to corticosteroids [7] [12].

Injections with HA have emerged as an alternative therapeutic strategy for diabetic patients or those who are refractory to or intolerant to conventional corticosteroids [1] [9]. In our study the effectiveness of HA injections was similar to corticosteroids for pain relief. In fact, the non-inferiority study demonstrated that the treatment with HA is non-inferior to triamcinolone acetonide after 6 months of follow-up. These results are in concordance with those from the only published study performed to date, i.e. using HA injected for treating trochanteric bursitis (although the journal where the manuscript was published is not indexed in JCR) [11]. This retrospec-

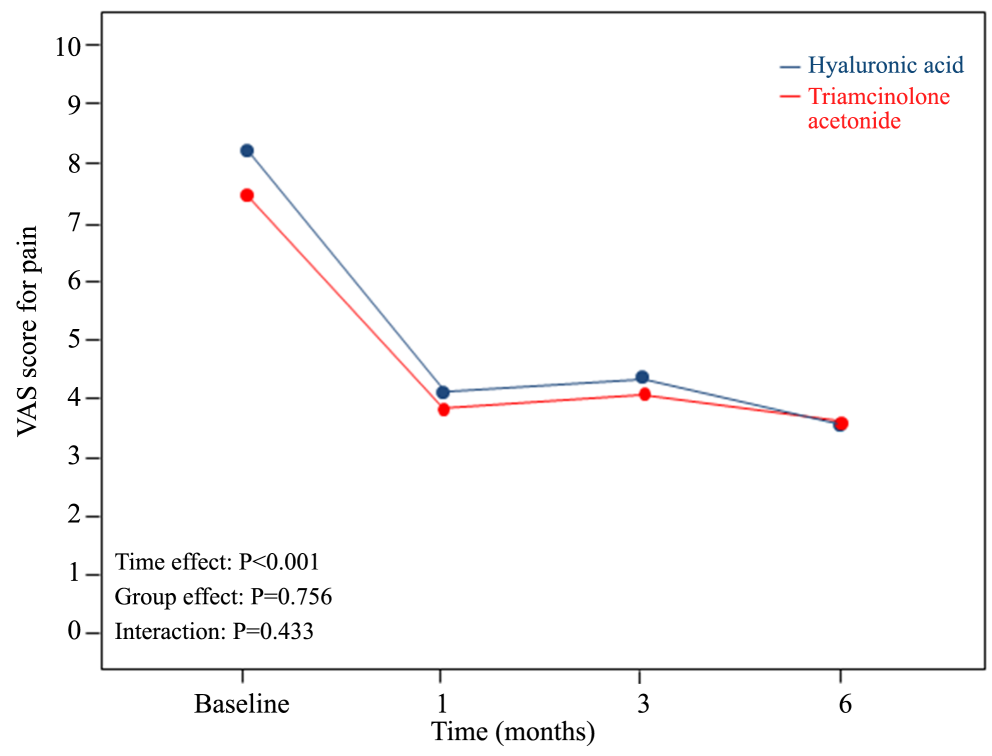

Figure 1. Evolution of VAS score for pain during the follow-up period between the two treatment groups. 


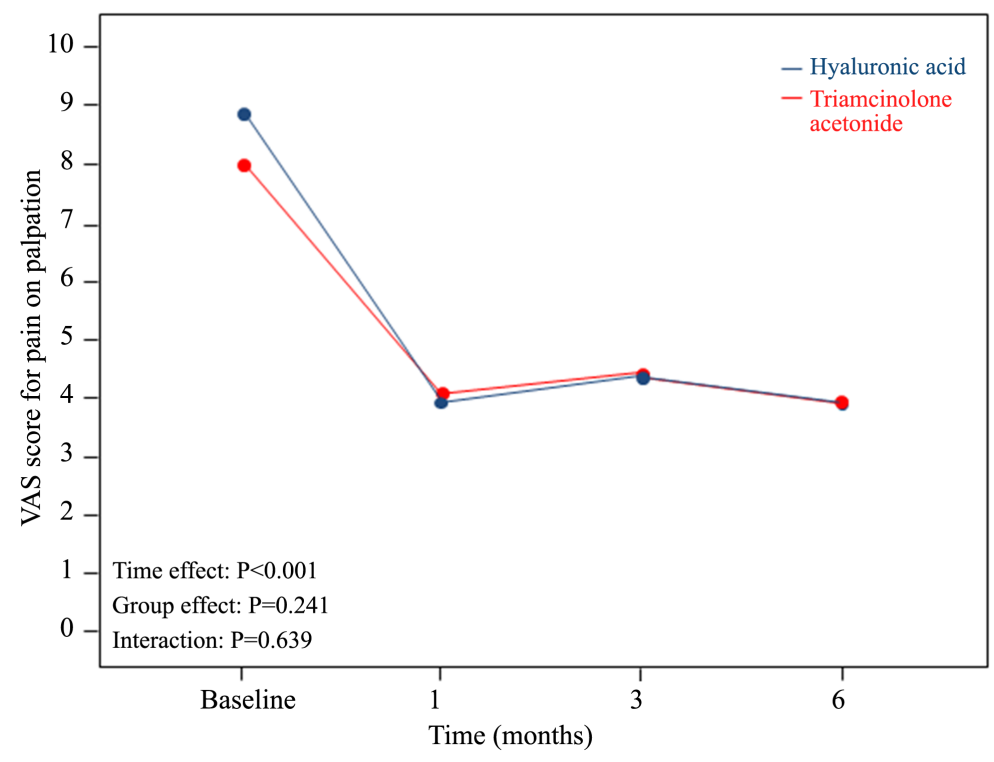

Figure 2. Evolution of VAS score for pain on palpation during the follow-up period between the two treatment groups.

tive study, that involved the follow-up of at least 12 months of 158 patients with trochanteric bursitis, showed the higher effectiveness of both the single injection of HA and its combination with corticosteroids than the conventional treatment with corticosteroids for treating trochanteric bursitis. Similarly, a study of 2009, evaluating the effects of corticosteroids in combination with HA injections for 3 months follow-up in 22 patients with scapulothoracic bursitis, showed a significant improvement in VAS for pain compared with baseline [13]. The effectiveness of HA has been also well established for the treatment of knee osteoarthritis, as demonstrated by rapidly reducing the pain and maintaining long-term effects [14]. Studies on knee osteoarthritis using intraarticular injections of HA recommend a dosage of per week, up to complete a total of 3 - 5 injections. However, for the treatment of scapulothoracic bursitis, authors used a single intra-bursal injection of $20 \mathrm{mg}$ high molecular-weight HA [13]. In our study, we used a single intra-bursal injection of $60 \mathrm{mg}$ high molecular-weight HA.

Injections with HA may also be an alternative for patients in whom the corticosteroid treatment failed to achieve a long-term benefit. In our study, the injections with HA showed that are effective and safe after 6 months of follow-up, producing stable pain relief over a long time without recurrence of symptoms, and an increase of the patient's satisfaction, as demonstrated by VAS for pain and for pain on palpation at the sixth month and the different Likert scales, respectively. Furthermore, no adverse events were reported during this period of time. These results achieved with HA may also lead to a reduction in the number of required arthroscopic bursectomies. Some limitations of the present study were the low number of patient included in the analysis, the lack of a controlled group with saline or a group consisting in the combination of corticosteroids and HA. Nevertheless, despite these limitations, results from our study are in concordance with similar literature. Further studies involving a large cohort of patients, additional treatment groups, and longer follow-up period are required to corroborate these results.

\section{Conclusion}

The treatment with HA has demonstrated to be non-inferior to corticosteroids after 6 months of follow-up in patients with GTPS. Therefore, the treatment with HA may be considered as an effective alternative therapeutic strategy for patients in whom the treatment with corticosteroids, or other therapies, have been unsuccessful or contraindicated, like the diabetic subjects.

\section{Conflict of Interests}

The authors declared no conflict of interests related to this work.

Mylan Institutional International provided the services of a professional medical writer but played no role in 
the study design or execution or analysis and interpretation of the data.

\section{Acknowledgements}

Authors would like to thank Pablo Vivanco (PhD, Meisys) for assisting in the preparation of the manuscript.

\section{References}

[1] Williams, B.S. and Cohen, S.P. (2009) Greater Trochanteric Pain Syndrome: A Review of Anatomy, Diagnosis and Treatment. Anesthesia Analgesia, 108, 1662-1670. http://dx.doi.org/10.1213/ane.0b013e31819d6562

[2] Segal, N.A., Felson, O.T., Torner, J.C., Zhu, Y.Y., Curtis, J.R., Niu, J.B. and Nevitt, M.C. (2007) Greater Trochanteric Pain Syndrome Epidemiology and Associated Factors. Archives of Physical Medicine and Rehabilitation, 88, 988-992. http://dx.doi.org/10.1016/j.apmr.2007.04.014

[3] Lievense, A., Bierma-Zeinstra, S., Schouten, B., Bohnen, A., Verhaar, J. and Koes, B. (2005) Prognosis of Trochanteric Pain in Primary Care. British Journal of General Practice, 55, 199-204.

[4] Gordon, E.J. (1961) Trochanteric Bursitis and Tendinitis. Clinical Orthopaedics, 20, 193-202.

[5] Ege Rasmussen, K.-J., Fano, N. (1985) Trochanteric Bursitis: Treatment by Corticosteroid Injection. Scandinavian Journal of Rheumatology, 14, 417-420. http://dx.doi.org/10.3109/03009748509102047

[6] Shbeeb, M.I., O’Duffy, J.D., Michet Jr, C.J., O’Fallon, W.M. and Matteson, E.L. (1996) Evaluation of Glucocorticosteroid Injection for the Treatment of Trochanteric Bursitis. Journal of Rheumatology, 23, 2104-2106.

[7] Cohen, S.P., Narvaez, J.C., Lebovits, A.H. and Stojanovic, M.P. (2005) Corticosteroid Injections for Trochanteric Bursitis: Is Fluoroscopy Necessary? A Pilot Study. British Journal of Anaesthesia, 94, 100-106. http://dx.doi.org/10.1093/bja/aei012

[8] Swezey, R.L. (1976) Pseudo-Radiculopathy in Subacute Trochanteric Bursitis of the Subgluteus Maximus Bursa. Archives of Physical Medicine and Rehabilitation, 57, 387-390.

[9] Habib, G.S. and Miari, W. (2011) The Effect of Intra-Articular Triamcinolone Preparations on Blood Glucose Levels in Diabetic Patients: A Controlled Study. Journal of Clinical Rheumatology, 17, 302-305. http://dx.doi.org/10.1097/RHU.0b013e31822acd7c

[10] Jordan, K.M., Arden, N.K., Doherty, M., Bannwarth, B., Bijlsma, J.W., Dieppe, P., et al. (2003) EULAR Recommendations 2003: An Evidence Based Approach to the Management of Knee Osteoarthritis: Report of a Task Force of the Standing Committee for International Clinical Studies Including Therapeutic Trials (ESCISIT). Annals of the Rheumatic Diseases, 62, 1145-1155. http://dx.doi.org/10.1136/ard.2003.011742

[11] Gorelick, L., Rozano-Gorelick, A., Robinson, D., Marcus, O., Joubran, S. and Ram, E. (2013) Treatment of Hip Trochanteric Bursitis Using Hyaluronate Injections. Open Journal of Rheumatology and Autoimmune Diseases, 3, 125-129. http://dx.doi.org/10.4236/ojra.2013.32019

[12] Walker, P., Kannangara, S., Bruce, W.J., Michael, D. and Van der Wall, H. (2007) Lateral Hip Pain: Does Imaging Predict Response to Localized Injection? Clinical Orthopaedics and Related Research, 457, 144-149.

[13] Chang, W.H., Im, S.H., Ryu, J.A., Lee, S.C. and Kim, J.S. (2009) The Effects of Scapulothoracic Bursa Injections in Patients with Scapular Pain: A Pilot Study. Archives of Physical Medicine and Rehabilitation, 90, 279-284. http://dx.doi.org/10.1016/j.apmr.2008.07.027

[14] Dixon, A.S., Jacoby, R.K., Berry, H. and Halmiton, E.B. (1988) Clinical Trial of Intraarticular Injection of Sodium Hyaluronate in Patients with Osteoarthritis of the Knee. Current Medical Research \& Opinion, 11, 205-213. http://dx.doi.org/10.1185/03007998809114237 